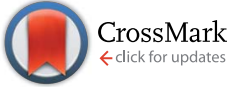

Cite this: Nanoscale, 2014, 6, 9632

Received 30th April 2014

Accepted 18th June 2014

DOI: $10.1039 / c 4 n r 02338 b$

www.rsc.org/nanoscale

\title{
Programmability of nanowire networks $\dagger$
}

\author{
A. T. Bellew, ${ }^{a b}$ A. P. Bell, ${ }^{a b}$ E. K. McCarthy, ${ }^{a b}$ J. A. Fairfield ${ }^{a b}$ and J. J. Boland ${ }^{\star a b}$
}

Electrical connectivity in networks of nanoscale junctions must be better understood if nanowire devices are to be scaled up from single wires to functional material systems. We show that the natural connectivity behaviour found in random nanowire networks presents a new paradigm for creating multifunctional, programmable materials. In devices made from networks of $\mathrm{Ni} / \mathrm{NiO}$ core-shell nanowires at different length scales, we discover the emergence of distinct behavioural regimes when networks are electrically stressed. We show that a small network, with few nanowire-nanowire junctions, acts as a unipolar resistive switch, demonstrating very high ON/OFF current ratios $\left(>10^{5}\right)$. However, large networks of nanowires distribute an applied bias across a large number of junctions, and thus respond not by switching but instead by evolving connectivity. We demonstrate that these emergent properties lead to fault-tolerant materials whose resistance may be tuned, and which are capable of adaptively reconfiguring under stress. By combining these two behavioural regimes, we demonstrate that the same nanowire network may be programmed to act both as a metallic interconnect, and a resistive switch device with high ON/OFF ratio. These results enable the fabrication of programmable, multi-functional materials from random nanowire networks.

\section{Introduction}

Random nanowire networks (NW networks) are of growing interest in the field of nanostructured electronic materials. Networks circumvent challenges associated with placement of single wires and natural property variations that occur from wire to wire. Applications of random networks include flexible, transparent conductors, ${ }^{1,2}$ artificial skin,,$^{3,4}$ and most recently biological sensing..$^{5,6}$ In the case of transparent conductors, current research has focused on oxide-free noble metal wires (e.g. $\mathrm{Ag}$ and $\mathrm{Au}$ ) that provide excellent connectivity and conductivity within networks. ${ }^{7-11}$ However, the high cost of these materials is expected to be a significant barrier to their widespread adoption, except in specific high-value niche applications.

Although there have been efforts to prevent or limit wire oxidation by the growth of core-shell structures,$^{12}$ an alternative strategy is to use the passivation layer itself as a means to control connectivity and hence conductivity within networks. Previously we demonstrated that it is possible to manipulate the connectivity within random networks comprised of polymer-

${ }^{a}$ School of Chemistry, Trinity College Dublin, Dublin 2, Ireland. E-mail: jboland@tcd.ie ${ }^{b}$ Centre for Research on Adaptive Nanostructures and Nanodevices (CRANN), Trinity College Dublin, Dublin 2, Ireland

$\dagger$ Electronic supplementary information (ESI) available: Nanowire statistics (length, diameter statistics, and oxide thickness) are provided. Forming curves for single junctions and networks. Passive voltage contrast image demonstrating selectivity of conductive pathways in $100 \mu \mathrm{m}$ network. See DOI: 10.1039/c4nr02338b coated $\mathrm{Ag}$ nanowires. ${ }^{13}$ In that case the evolution of the connectivity and subsequent growth in network conductivity is controllable but irreversible, because connectivity evolution involves the irreversible dielectric breakdown of the passivating polymer so that the network cannot be turned off. In this work we employ inexpensive reactive metal nanowires and exploit the phenomenon of resistive switching (RS) in the passivating metal oxide layer. RS is a well-known reversible switching phenomenon that is the basis for resistive random access memory (RRAM) ${ }^{14-18}$ and its incorporation into nanowire networks allows us to examine the effect of junction reversibility.

Recently, Stieg and Gimzewski et al. have reported on similar work, studying the adaptive behaviour displayed by memristive networks of atomic switches. ${ }^{19-23}$ They demonstrate the analogous behaviour of network systems based on the bipolar Ag/ $\mathrm{Ag}_{2} \mathrm{~S} / \mathrm{Ag}$ junction to those of the complex synaptic network in the human brain. It was found that the behaviour of these memristive systems stems from the interactions of all junctions in the network, creating a global response. ${ }^{20}$ We shall demonstrate that the behaviour of Ni nanowire networks are governed by the unipolar NiO junctions. From this localized, scale dependent properties emerge that introduce the ability to program these otherwise random systems.

Planar metal-NiO-metal device structures are well known to display RS properties, ${ }^{24,25}$ and recent results by He et al. ${ }^{26}$ and Cagli et al. $^{27}$ demonstrate RS in both single and crossbarstructured $\mathrm{Ni} / \mathrm{NiO}$ core-shell nanowires. In the latter case $\mathrm{NiO}$ acts as the active switching material, while the Ni core plays the role of the contact metal electrodes. The results by Cagli et al. are important as they demonstrate the ability of a single 
NW-NW junction to act as a resistive switch. Whether a particular network junction undergoes RS depends on the local electric field experienced by the junction, the current flowing though the junction and hence on the status of neighbouring junctions. Here we demonstrate that the sensitivity of individual junctions to non-local inputs defines a natural length scale within the network, and ultimately allows the network to be programmed to exhibit a range of material and device behaviours. Specifically we show that at length scales below a certain threshold, a random network behaves as a high performance RS memory device, while at longer length scales the same network exhibits the properties of a fault-tolerant conducting film capable of adaptively reconfiguring under electrical stress. Finally, we demonstrate that devices defined within the network can be driven through the network itself, so that the network can be programmed to simultaneously operate as the device channel and interconnect material.

\section{Experimental details}

Ni NWs were purchased from Nanomaterials.it. Each Ni wire is coated by a self-limiting NiO layer $4-8 \mathrm{~nm}$ in thickness under ambient conditions, and the average length (diameter) of the nanowires was $10.6 \mu \mathrm{m}(81 \mathrm{~nm}$ ) (see Fig. S2†). Single NWs were contacted by drop-casting a $\mathrm{NW}$ /isopropanol dispersion onto clean $300 \mathrm{~nm}$ thermal $\mathrm{SiO}_{2}$ with pre-defined $\mathrm{Au}$ contact pads. Electron beam lithography (EBL) followed by metal evaporation was used to contact individual nanowires. Crossed-nanowires were contacted through a magnetic alignment technique previously described, in combination with EBL. ${ }^{27}$ Nanowire networks were formed by spray depositing the nanowire dispersion onto clean $\mathrm{SiO}_{2} \cdot{ }^{28}$ Metal contact electrodes were deposited by electron beam evaporation through a shadow mask. Ni was used as a contact metal for all devices. Electrical characterisation was performed with a Keithley 4200 SCS. The Zeiss Ultra Plus scanning electron microscope, and the FEI Titan transmission electron microscope were used for all microscopy.

\section{Results and discussion}

We begin by studying the behaviour of individual junctions and then examine the behaviour as the numbers of junctions are increased to form networks of different sizes. Fig. 1(a) shows two independently contacted Ni NWs that meet to form a single junction. It is well established that $\mathrm{RS}$ in $\mathrm{NiO}$ involves the formation of a conductive filament through the insulating oxide layer between two metal contacts. ${ }^{\mathbf{1 4}}$ The junction in Fig. 1(a) is initially in a non-conducting, pristine state $(R=1.7 \mathrm{M} \Omega)$. Following initial threshold forming sweeps to $100 \mathrm{nA}$ and $1 \mu \mathrm{A}$ compliance current respectively (see ESI $\dagger$ ), a current-voltage (I$V$ ) sweep is performed and the device reaches a compliance current of $10 \mu \mathrm{A}$ at $2.8 \mathrm{~V}$ (Fig. 2(a), red trace); a subsequent $I-V$ demonstrates that the junction has become ohmic and is in a low resistance state (LRS) $(R=3.2 \mathrm{k} \Omega)$. In this case conductive filament formation occurs in response to the applied electric field across the single junction, which may be thermally
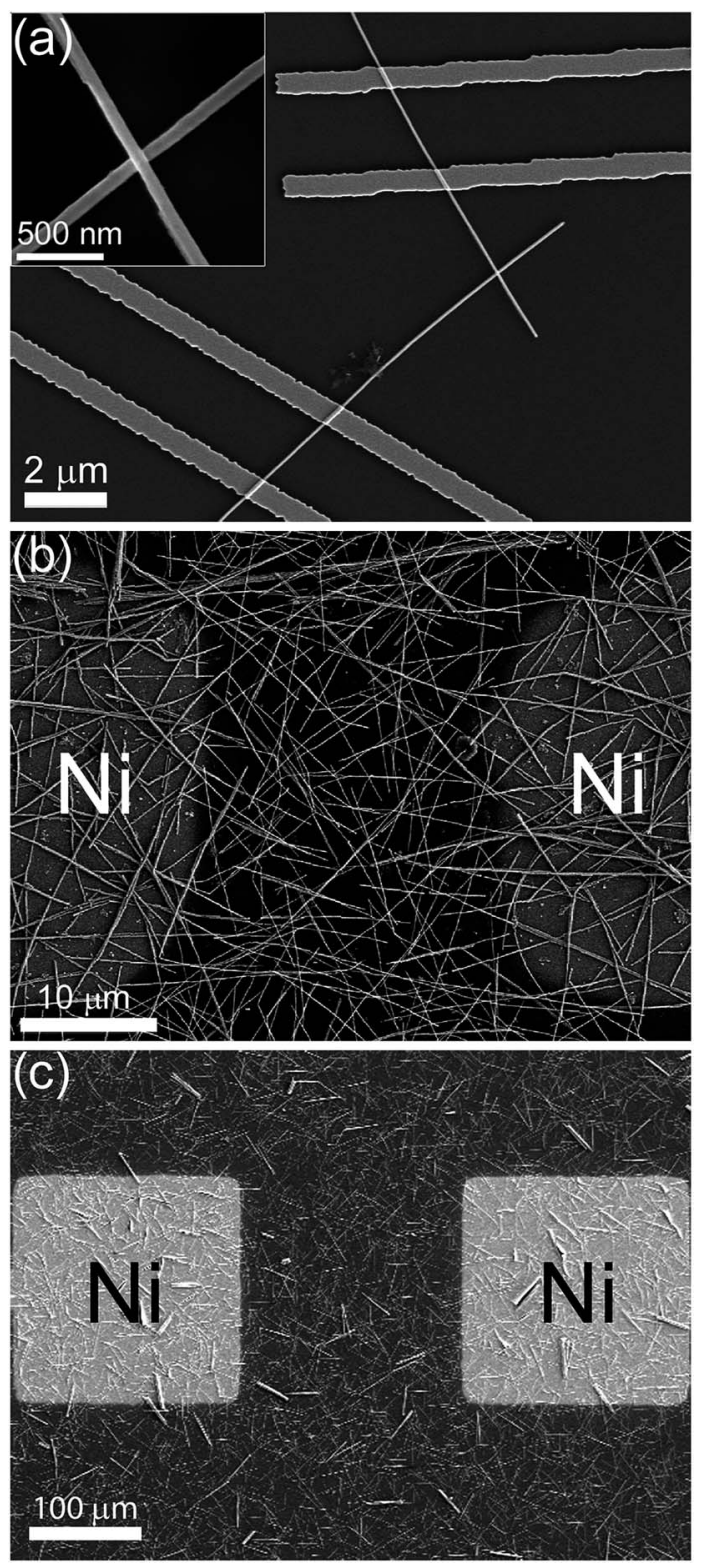

Fig. 1 Ni nanowires and NW networks. (a) Two nanowires cross to form a single junction, and are subsequently contacted with Ni metal lines. A magnified image of the junction is shown in the inset. (b) and (c) Nanowire networks of size, $D / L_{\mathrm{NW}}=1.88$ and 9.43 respectively formed between two Ni contact electrodes. Nanowire density is $\rho=0.38 \mathrm{NW}$ $\mu \mathrm{m}^{-2}$. Here $W=D$ to create a square electrode geometry.

disrupted by Joule heating when the current exceeds a value, $I_{\text {RESET }}$, at a voltage, $V_{\text {RESET }}$, causing the device to revert to a high resistance state (HRS) as shown in Fig. 2(a), consistent with unipolar RS operation. ${ }^{29-31}$ During the forming and set processes a compliance current level of $10 \mu \mathrm{A}$ is set to ensure formation of a strong conductive filament while preventing the device from switching back to the HRS at zero bias, a phenomenon known as threshold switching. ${ }^{32}$

Currently the fabrication of these single junction structures is a complex multistep process and the yield of viable devices is invariably low. In comparison, the fabrication of random 

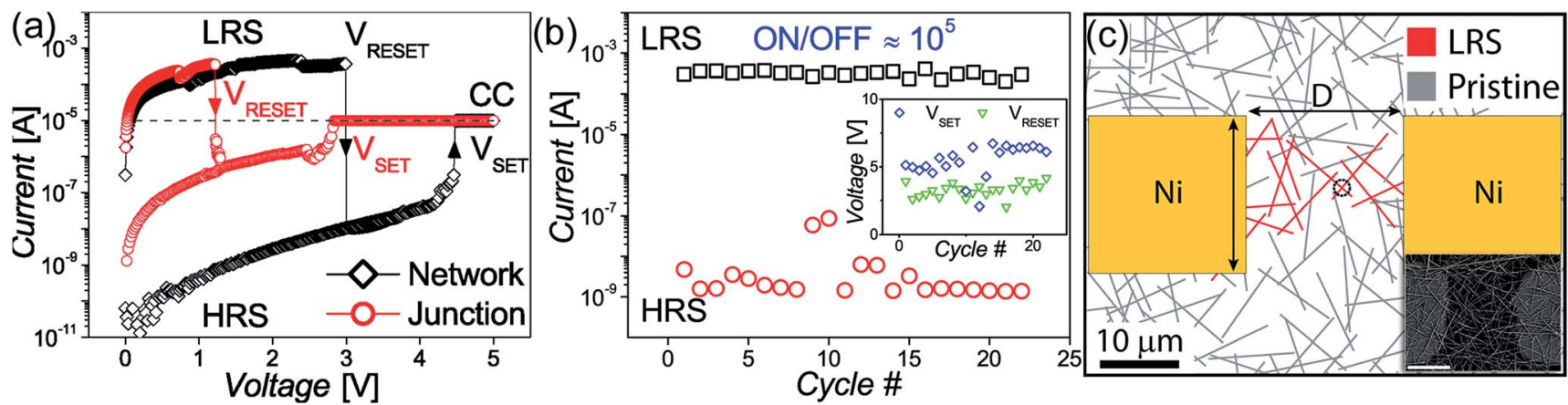

Fig. 2 Resistive switching in Ni NW networks. (a) Crossed nanowires (red open circles) and NW networks (black open diamonds) with small D/ $L_{\text {NW }}(1.88)$ both operate as RS devices, where the application of an electric field switches the device from a HRS to a LRS, and vice versa. Variations in the HRS are common for RS systems such as these (see (b)), and is the cause of the increased $V_{\mathrm{SET}}$ value for the network device. An increase in path length accounts for the higher resistance of the network LRS, and hence the larger $V_{\text {RESET }}$ voltage. The $I_{\text {RESET, }}$, however, is almost identical for both systems. Voltage sweep rates are $\sim 50 \mathrm{mV} \mathrm{s}^{-1}$. (b) An ON/OFF ratio (read voltage $=1.5 \mathrm{~V}$ ) of $10^{5}$ is shown for one such network device. The nanoscale contact area at the junctions reduces the leakage current and is thought to be the reason behind these very high values. The mechanism proposed for RS in networks is schematically illustrated in (c), for a network where $D / L_{\mathrm{NW}}=2$. Small networks form cells of NWs connected by conductive filaments at the NW-NW junction. The connectivity cell (depicted as red wires) spans the electrodes, but is reliant on a single junction (circled). This junction, if stressed, will switch and produce the RS behaviour seen in (a).

networks, such as that shown in Fig. 1(b), is readily scalable and involves only a two-step procedure. ${ }^{28}$ As we shall show, by taking advantage of the natural connectivity properties of random networks we demonstrate RS behaviour comparable to that of a single junction outlined above but without the same fabrication challenges. We define a network size, $D / L_{\mathrm{NW}}$, by depositing metal contact electrodes, where $D$ is the interelectrode distance, and $L_{\mathrm{NW}}$ the average nanowire length (see Fig. $\mathrm{S} 2 \dagger$ ). The electrode width, $W$, is equal to $D$ unless otherwise specified. Fig. 1(b) and (c) show networks of size $D / L_{\mathrm{NW}}=1.88$ and 9.43, respec-

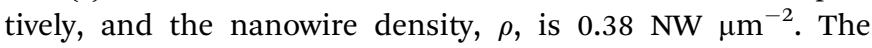
nanowire density was kept constant for all data presented, unless otherwise specified.

\section{Resistive switching in small networks $\left(D / L_{\mathrm{NW}}<2\right)$}

We demonstrate the operation of a Ni NW network similar to that shown in Fig. 1(b) $\left(D / L_{\mathrm{NW}}=1.88\right)$ as a RS in Fig. 2(a) (black open diamonds). The initial forming step involves the formation of connectivity cells that ultimately connect together to establish a continuous conducting path between the two electrodes. ${ }^{13}$ The conducting path formed will be comprised of some number of wires and connecting junctions each capable of resistive switching. For small electrode separations $\left(D / L_{\mathrm{NW}} \sim\right.$ 1) we showed previously that the majority of the wires between the electrodes are activated during the forming step, ${ }^{13}$ but at larger separations $\left(D / L_{\mathrm{NW}}>1\right)$ the network selects those junctions that are easiest to activate when forming the LRS conducting path that initially bridges the electrodes, and which may then be operated as a RS device. A passive voltage contrast image demonstrating this selectivity is included in the ESI ( $\mathrm{S} 2 \dagger)$. The mechanism we propose for RS in random networks is schematically illustrated in Fig. 2(c). A network of size $D / L_{\mathrm{NW}}=$ 2 has undergone a forming step and is in a LRS; although multiple wires contribute to the LRS (indicated by red wires), the connectivity within the network is essentially percolative in nature and is reliant upon a single junction (circled) to remain in the LRS state. We propose that junctions like this form bottleneck points for the current, and are susceptible to switching when electrically stressed.

Comparing the single junction and $\mathrm{Ni}$ NW network in Fig. 2(a), we note the RS properties remain largely the same. Large variations in the HRS from cycle to cycle are common in nanowire RS systems such as these, ${ }^{33}$ with ranges approaching $10^{3}$ reported elsewhere. ${ }^{27}$ These variations account for the differences observed in $V_{\text {SET }}$ values, while the increase in $V_{\text {RESET }}$ is due to the added resistance associated with an increased path length. However, we see that $I_{\text {RESET }}$ occurs at almost identical values for both the single junction and NW network (348 $\mu \mathrm{A}$ and $366 \mu \mathrm{A}$, respectively). This implies that it is indeed a single junction that is responsible for the switching observed in both cases. We will explore this idea further in Fig. 4.

An ON/OFF current ratio of $>10^{5}$ is shown in Fig. 2(b) over 20 cycles of the NW network device operation. The ON/OFF ratio of any RS device is controlled by the device cross-sectional area and determines the leakage current in the HRS. For a network, this area is defined by the two crossed NWs that undergo RS switching. The nanoscale nature of this contact leads to minimal leakage current, and thus is responsible for the exceptionally high ratios seen in network devices. While no definitive conclusions can be drawn at this point, the high fidelity of the current levels in the ON state indicates that the same set of junctions are involved in establishing the LRS, hence the properties of the conductive filaments involved do not vary significantly between cycles.

\section{Scaling of $V_{\text {FORM }}$}

As mentioned above, before RS behaviour can be established a connectivity pathway through the network must first be formed. How the forming voltage, $V_{\text {FORM }}$, scales is important, as it will ultimately limit the addressable network size. A plot of $V_{\text {FORM }}$ 
against $D / L_{\mathrm{NW}}$ is shown in Fig. 3 for three different nanowire

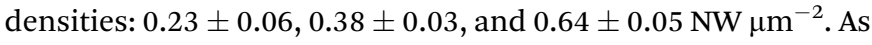
the network size increases, a greater number of junctions are active in the formed conductive pathway, hence we observe a linear scaling of $V_{\text {FORM }}$ with $D / L_{\mathrm{NW}}$ for each series. We note that the strong linear dependence demonstrated here implies the formation of conductivity pathways largely confined to the area defined by the electrodes, despite a practically infinite source of nanowires outside of this area. The slope becomes less steep as the NW density increases, with the $0.38 \mathrm{NW} \mu \mathrm{m}^{-2}$ sample displaying a slope of $3.66 \mathrm{~V}$ per nanowire length. This is consistent with the presence of a larger density of junctions as the NW density increases, which increases the probability of finding a set of lower barrier junctions that span the network, hence reducing $V_{\text {FORM }}$, consistent with results previously reported. ${ }^{13}$ However, high voltages are required to activate networks larger than $D / L_{\mathrm{NW}}=50$. This is potentially a serious limitation to the future development of NW networks as materials and devices, and we are currently looking into strategies to reduce $V_{\text {FORM }}$, as well as alternate methods of activating large networks of nanowires with resistive junctions.

\section{Resistive switching and probability in networks $\left(D / L_{\mathrm{NW}}<10\right)$}

The repeatable, device-like behaviour demonstrated in Fig. 2 can also be demonstrated for networks as large as $D / L_{\mathrm{NW}}=9.43$.

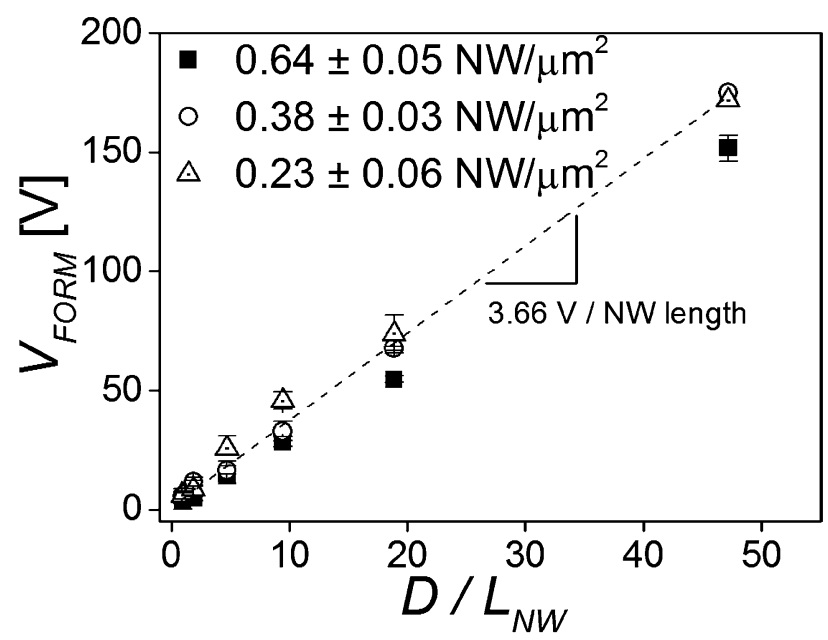

Fig. 3 Scaling of $V_{\text {FORM }}$ with $D / L_{N W}$ for different network densities:

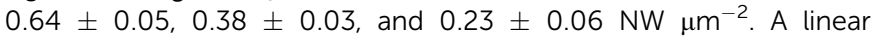
dependence of $V_{\mathrm{FORM}}$ on network size is apparent in each case, with the $0.38 \mathrm{NW} \mu \mathrm{m}^{-2}$ series displaying a slope of $3.66 \mathrm{~V}$ per nanowire length. The slope also shows a dependence on network density. Due to the high voltages required, we are limited to networks of size $D / L_{N W}$ $\leq 50\left(V_{\text {MAX }}= \pm 210 \mathrm{~V}\right)$. We are currently looking into methods to reduce $V_{\text {FORM }}$, as well as alternate strategies to activation, in order to overcome this limitation. Each data point here represents a mean value for that network size, while the error bars represent the standard deviation of the values. The sweep rate ranged from $50 \mathrm{mV} \mathrm{s}^{-1}$ at $D /$ $L_{\mathrm{NW}}=1$ to $5 \mathrm{~V} \mathrm{~s}^{-1}$ at $D / L_{\mathrm{NW}}=50$. Despite the varying sweep rate, no dependence on sweep rate is observed, as should be indicated by a change in slope. NW density measurements were made across multiple $10 \times 10 \mu \mathrm{m}$ areas; the error values represent the standard deviation of these measurements.
Fig. 4 compares the RS operation parameters $V_{\mathrm{RESET}}, V_{\mathrm{SET}}$, and $I_{\text {RESET }}$ for a number of devices and a range of network sizes alongside those of a single junction $\left(D / L_{\mathrm{NW}}=0\right)$. We note both $V_{\text {RESET }}$ and $V_{\text {SET }}$ increase with network size, consistent with the larger applied voltage necessary to create the required voltage drop across the individual junctions that comprise the switching network. These voltage levels are much smaller than required during network forming (see Fig. 3), consistent with the hypothesis that the vast majority of wires contributing to the LRS remain unaffected by the switching event. The voltage drop is then largely across the switching junction, with only a small contribution falling across the rest of the network. In contrast, during the forming operation there is a significant voltage drop across all the junctions that ultimately connect together to form the LRS. These conclusions are confirmed by the observed independence of $I_{\text {RESET }}$ on network size, as shown in Fig. 4. In the absence of parallel paths, the failure of a single junction is sufficient to switch the network from the LRS to the HRS. Since these junctions are essentially in series this precludes the resetting of multiple junctions along the path, hence the near constant $I_{\text {RESET }}$ level in Fig. 4. Therefore for larger networks despite the large numbers of junctions involved, the RS phenomenon still involves the switching of a single junction
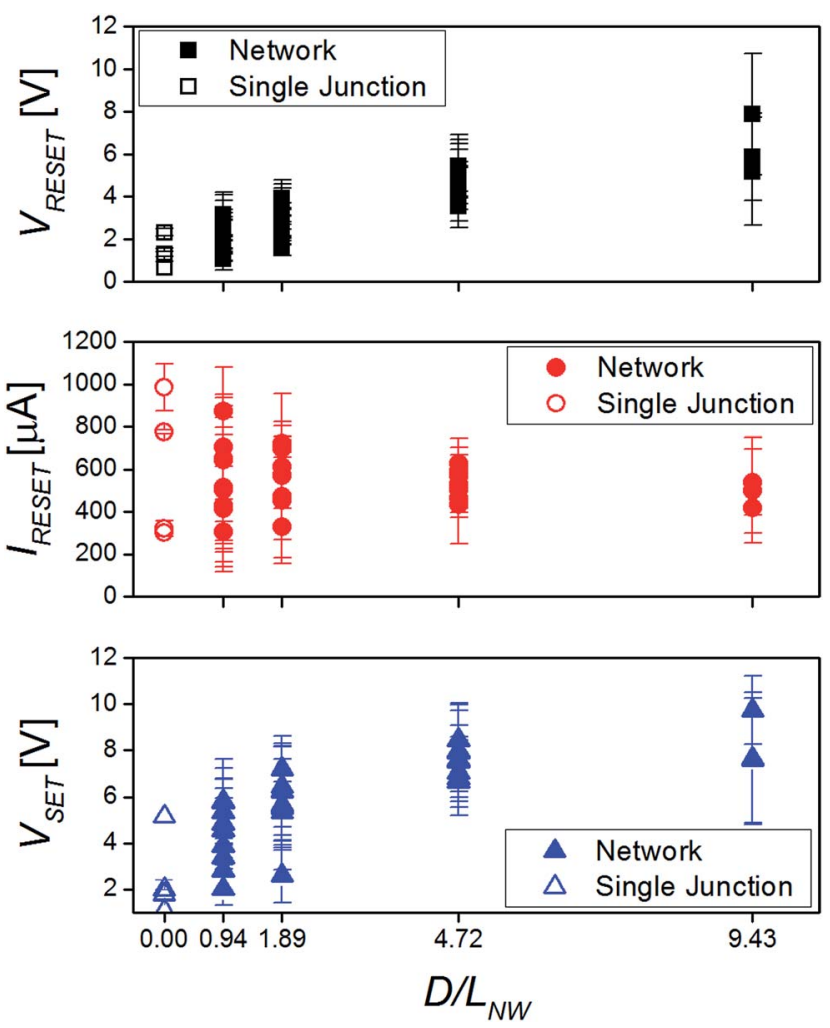

Fig. 4 Scaling of RS in networks. RS parameters $V_{\text {RESET, }} I_{\text {RESET, }}$ and $V_{\text {SET }}$ and are shown for multiple devices at various network sizes. Each data point represents the mean value for a single device, while the error bars show the standard deviation. A clear dependence on network size is seen for both $V_{\text {RESET }}$ and $V_{\text {SET }}$, but not for $I_{\text {RESET }}$. Given the similar values of $I_{\text {RESET }}$ for networks and single junctions, it can be concluded that a single junction is responsible for the switching behaviour seen, even in larger networks. Voltage sweep rate was $\sim 50 \mathrm{mV} \mathrm{s}^{-1}$. 
along the conducting path, and is thus responsible for the high ON/OFF ratio and the high fidelity of the ON (LRS) and OFF (HRS) states. Although the RS parameter data presented in Fig. 4 displays a significant spread in values, it is no higher than that seen for single junction measurements (open symbols, Fig. 4). Again, this variability is inherent to all RS devices, likely compounded in this case by the variability of the junction interface, the main source of which is the variance in oxide thickness from wire to wire (see Fig. S1.1†).

As the network size is increased beyond $D / L_{\mathrm{NW}}=5$, the tendency of junctions to undergo resistive switching reduces significantly. We previously demonstrated that the degree to which a polymer-coated Ag NW network can evolve connectivity increases as a function of network size. ${ }^{13}$ In larger Ni networks there are significant numbers of junctions that are not connected to the conducting paths within the network (see Fig. S3.1†) and the network responds to the increasing levels of electrical stress by connecting these junctions rather than causing junctions within the conducting path to undergo RS. This behaviour reflects the intrinsic level of redundancy that exists in larger networks and becomes more pronounced at higher NW densities, as illustrated in Fig. 5 where we have plotted the probability of switching, $P_{\mathrm{RS}}$, against network size. Here, we define the RS probability as:

$$
P_{\mathrm{RS}}=\frac{n(\mathrm{RS})}{n(T)}
$$

where $n(T)$ is the number of samples measured, and $n(\mathrm{RS})$ is the number of samples where RS was observed. A device is defined as being capable of RS if it demonstrates at least two complete RS cycles (LRS $\rightarrow$ HRS $\rightarrow$ LRS $\rightarrow$ HRS), and RS parameters fall in the ranges shown in Fig. 4. This definition is not intended to be compared with the state-of-the-art performance, but rather as a means to categorize behaviour within these network systems.

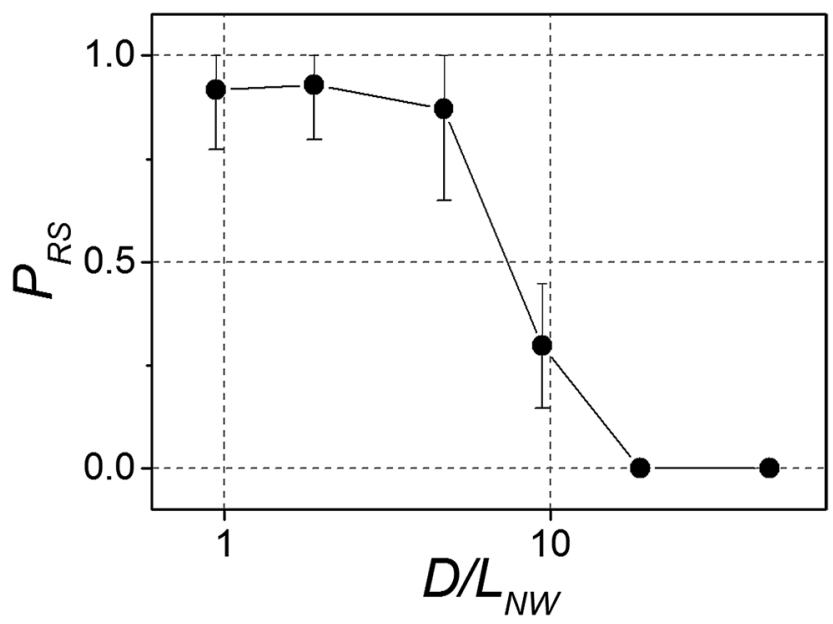

Fig. 5 Plot of switching probability, $P_{\mathrm{RS}}$, against network size, $D / L_{\mathrm{NW}}$ (log scale). A sharp decrease in switching probability is seen at $D / L_{\mathrm{NW}}=$ 4.7. Beyond this point, the capacity of the network to increase its connectivity begins to outweigh the tendency towards RS switching behaviour. Voltage sweep rate was $\sim 50 \mathrm{mV} \mathrm{s}^{-1}$ for all data.
The initial conducting path (LRS) that is established following the formation process is essentially a percolative connectivity path that spans the network between the two electrodes. When $D / L_{\mathrm{NW}} \sim 1$ the majority of the junctions between the electrodes participate in the percolative pathway, so when the network is electrically stressed there is a strong tendency to undergo RS. The failure of a single junction within the percolative connectivity path causes the network to switch to the HRS. However, in the case of a large network $\left(D / L_{\mathrm{NW}} \gg 1\right)$ there remain within the area of network defined by the electrodes many junctions that are not connected to the conducting path (see Fig. S3.1†). Under electrical stressing these networks tend to add additional junctions to the connectivity path, thereby densifying the conducting pathway within the network. Consequently, the failure of any one junction within this path has an increasingly reduced probability of causing the network to switch between the LRS and HRS, consistent with the data in Fig. 5.

\section{Large networks $\left(D / L_{\mathrm{NW}}>10\right)$}

The capacity of larger networks to evolve or add additional junctions is illustrated in Fig. 6 where we show the hysteretic electrical behaviour of a network of size $D / L_{\mathrm{NW}}=9.43$. As the voltage is swept we see significant levels of hysteresis in the $I-V$ curves, similar to that observed for PVP-coated Ag NW networks. ${ }^{13}$ Hysteresis occurs because at higher voltages additional junctions are switched ON extending the conducting pathway and increasing the overall conductivity of the network

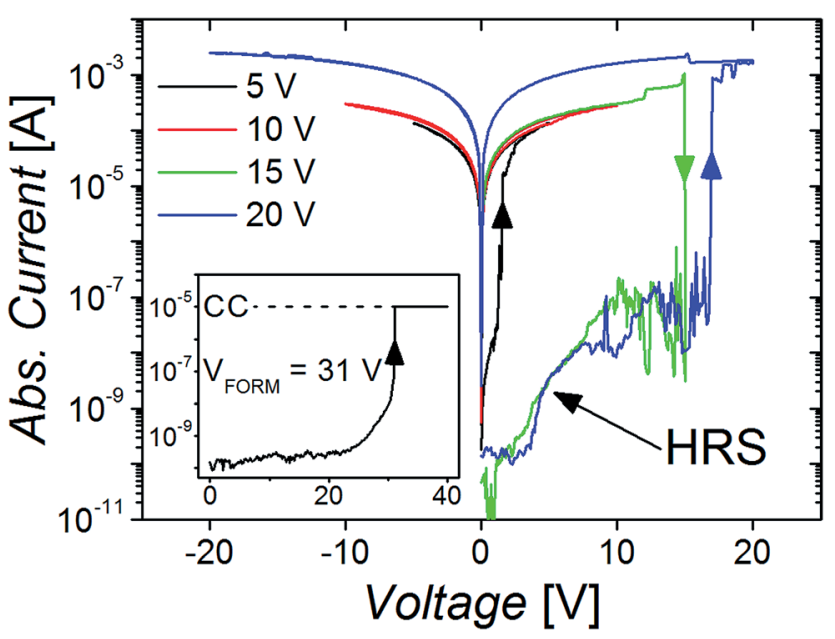

Fig. 6 RS and evolving connectivity behaviour in larger NW networks. Intermediate network sizes $\left(D / L_{\mathrm{NW}}=9.43\right)$ evolve their connectivity through the addition of nanowires to existing conductive paths, indicated by hysteretic behaviour in the $I-V$ curves (see black trace). Due to the greater number of junctions to be formed, $V_{\mathrm{FORM}}$ scales with the electrode separation (inset). At this network size, $P_{\mathrm{RS}}$ is non-zero and thus RS behaviour is still observed; the network switches from a LRS to a HRS (green trace) as a critical junction is reset. A subsequent sweep recovers the LRS (blue trace), and the network evolves even further to achieve a final resistance of $5.6 \mathrm{k} \Omega$. Networks of this size thus display a hybrid behaviour between that of a device and a material. Voltage sweep rates ranged from $125 \mathrm{mV} \mathrm{s}^{-1}$ at $5 \mathrm{~V}$, to $500 \mathrm{mV} \mathrm{s}^{-1}$ at $20 \mathrm{~V}$. 
LRS (see black trace, Fig. 6). Consequently, much higher voltages may be applied without the network switching back to the HRS. Moreover, the conductivity of the network is exceptionally stable at low biases; the average voltage across any one junction is insufficient to affect its state, hence the distribution of ON and OFF junctions is fixed. For a network of this size, while $P_{\mathrm{RS}}$ is significantly less than unity it remains finite, as seen by the green trace in Fig. 6. The network is observed to suddenly jump to the HRS at an $I_{\text {RESET }}$ value of $1.1 \mathrm{~mA}$. We propose that at this point the voltage required to evolve the network further was greater than that required to reset the weakest junction in the dominant conducting path. This, however, is a reversible phenomenon as can be seen in the subsequent $I-V$ sweeps. The network switched to a LRS at $17 \mathrm{~V}$ (blue trace), much lower than $V_{\mathrm{FORM}}$, and consistent with our earlier conclusion that the vast majority of the conducting path remains unaffected by the prior switching event. Following the re-establishment of the LRS the network was able to further evolve connectivity to reach a final resistance of 5.6 $\mathrm{k} \Omega$. The behaviour of a network of this size can thus be thought of as a hybrid of a RS and an evolutionary material.

By extending the scale of the network beyond $D / L_{\mathrm{NW}}=10$ we can eliminate the propensity of the network to undergo RS, thereby creating an adaptive material, capable of reconfiguring under stress. Fig. 7 shows electrical characteristics of a large network $\left(D / L_{\mathrm{NW}}=47.17\right)$. It is clear that the larger network displays more pronounced hysteretic behaviour, such that it is possible to precisely control the resistance of the network (Fig. 7(a), left inset). This controllability is directly related to the size and density of the network, as additional junctions are continuously added to established conducting paths at higher applied voltages. The network connectivity typically evolves in a smooth fashion that depends on the applied voltage and sweep rate. The larger hysteresis loops observed at positive bias are a result of the voltage sweep direction: $0 \rightarrow+V_{\max } \rightarrow 0 \rightarrow-V_{\max }$ $\rightarrow 0$ and are consistent with unipolar RS being dependent on the magnitude, but not the direction, of the applied field. However sudden large jumps in current can also be observed (see arrow, Fig. 7(a)) especially in the earliest stages of activation as large sections of network are added to established conducting paths. This behaviour is very similar to the avalanche breakdown mechanism used to describe the growth of percolating clusters in random circuit breaker models. ${ }^{34}$

The level of connectivity in large networks is so extensive that it is not possible to switch the network to a HRS even with very high applied voltages. Localised RS switching within the network still occurs, but the resulting changes in network connectivity induced by any localised switching events results in only a modest and temporary reduction in conductivity. The inherent redundancy within these large networks enables them to spontaneously program or reconfigure by activating alternate connectivity pathways or reactivating junctions that had previously been reset. An enlarged view of the $I-V$ curve in Fig. 7(b) shows the conductance of the network decreasing abruptly only to jump to a new higher conductance level seconds later. This dynamic reconfigurability in response to an electrical stress is a characteristic of these networks and continues until a stable conductive path is established.
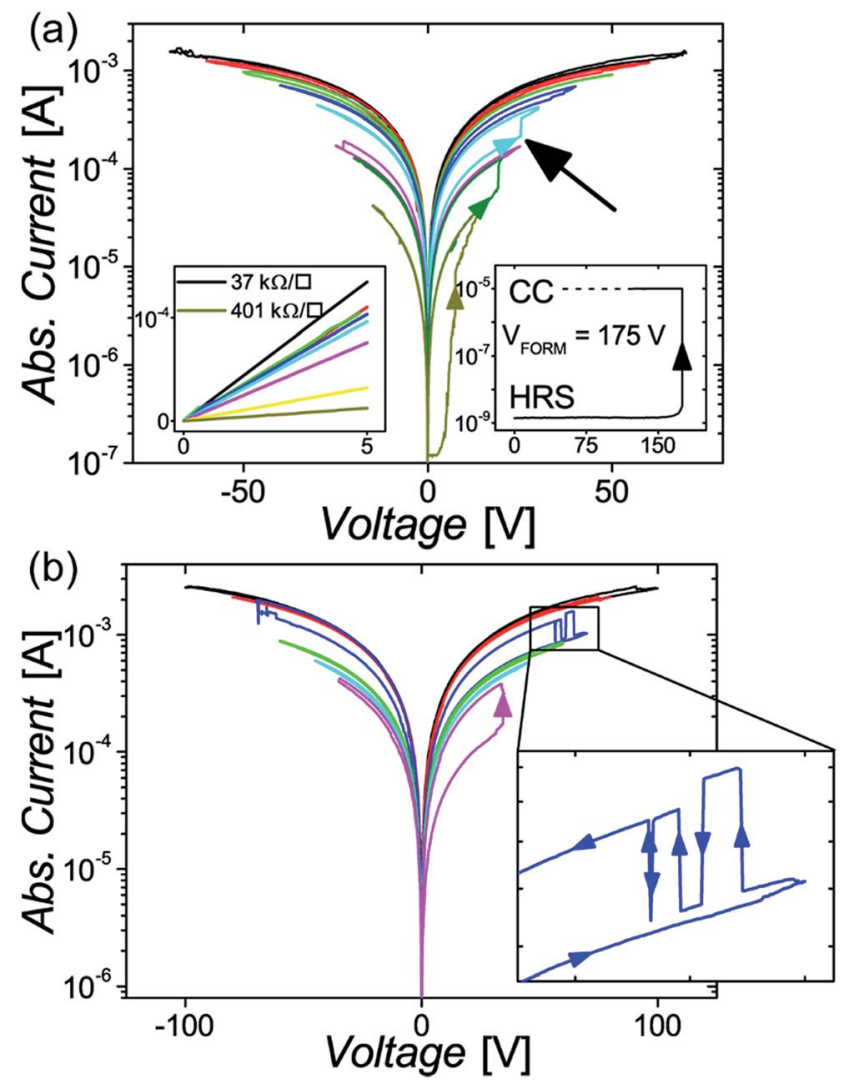

Fig. 7 Hysteretic and adaptive nature of very large $\left(D / L_{N W}=47.17\right)$ networks. (a) Very large networks provide numerous pathways to current flow, such that the connectivity may be evolved with a high degree of control, and the resistance is tuneable over a wide range (a, left inset). The high forming voltage reflects the larger numbers of junctions that have to be activated to establish a conducting path. (b) Large networks also display adaptive reconfiguration of the current paths under high stress. The resistance of the network may increase at extreme current levels as individual junctions reset, however redundancy within the network prevents the whole network from resetting (b, inset). Sweep rates ranged from $25 \mathrm{mV} \mathrm{s}^{-1}$ at $5 \mathrm{~V}$ to $250 \mathrm{mV} \mathrm{s}^{-1}$ at $100 \mathrm{~V}$.

\section{Programming networks}

The scale dependent behaviour of networks reported here, including the ability to dynamically respond to electrical stressing, suggests the possibility of programming the network to perform various functions. One example of this is demonstrated in Fig. 8. Fig. 8(a) shows two network sizes, $D / L_{\mathrm{NW}}=0.94$ and 9.43 respectively, defined by electrodes I-III. The RS properties of the network defined across I-II are shown in Fig. 8(b) (black trace). The forming voltage of $12 \mathrm{~V}$ establishes a LRS, which in turn can be repeatedly switched to the HRS at a $V_{\text {RESET }}$ of 7 V. Fig. 8(c) shows the resulting $I-V$ curve after the network defined across II-III has been evolved so that is behaves as a stable ohmic conductor with a resistance of $29 \mathrm{k} \Omega$. The conduction properties of this network region are stable over long periods of time in ambient conditions. The inset in Fig. 8(c) shows no loss of conductivity over an 80 day test period, indicating that the conducing filament within the oxide is not susceptible to oxidation. 

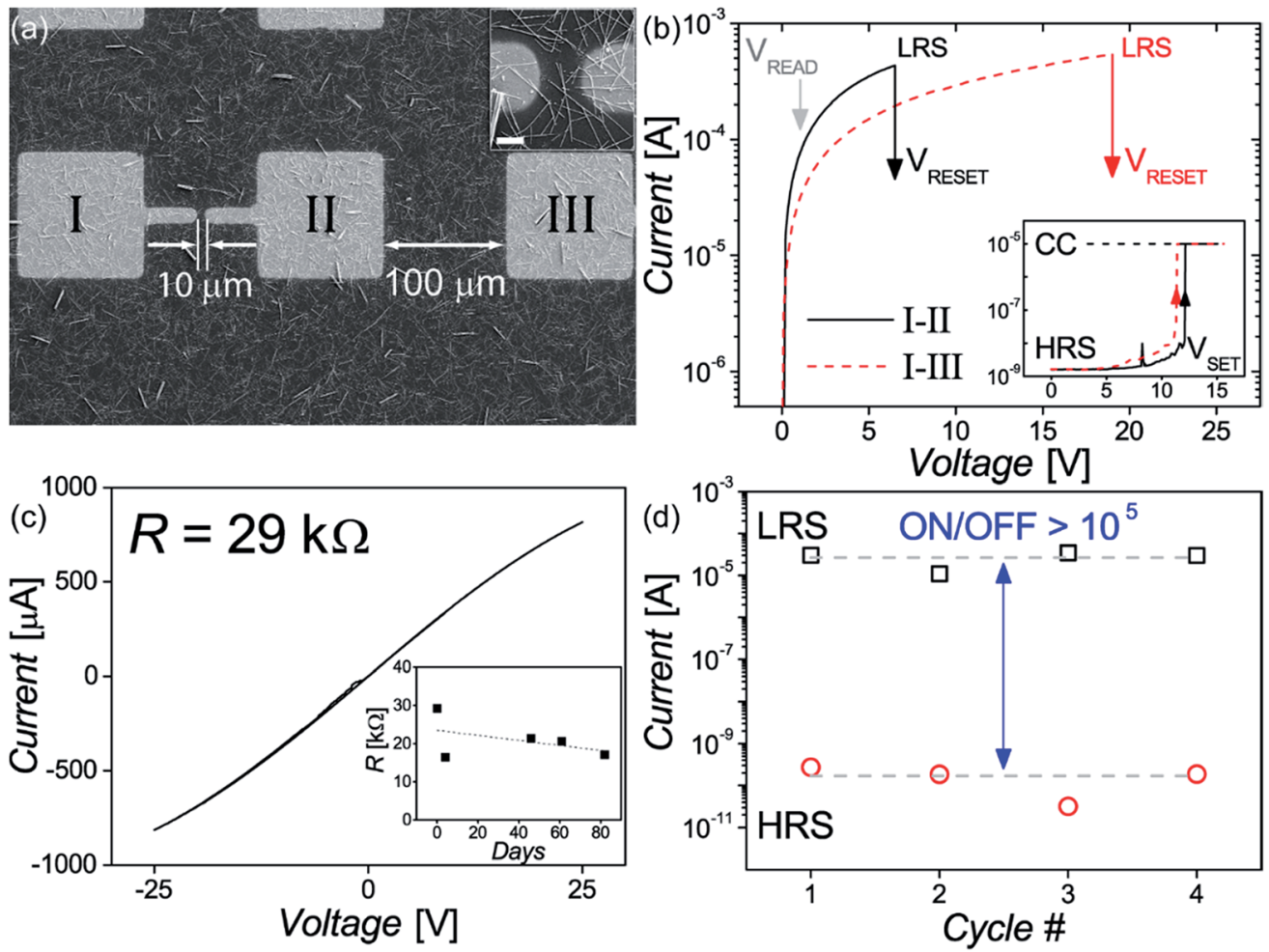

Fig. 8 Programmability of transparent Ni NW networks. (a) Ni contact pads define two network sizes (0.94 and 9.43) on a sparse network ( $\rho=$

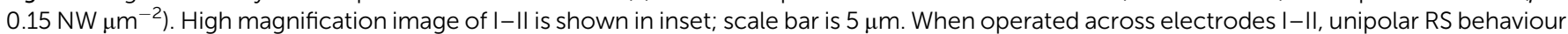
is observed (b, black trace). (c) The network defined by electrode II-III demonstrates ohmic behaviour, showing a resistance of $29 \mathrm{k} \Omega$, after it has been electrically stressed $( \pm 25 \mathrm{~V})$. This conduction state is highly stable, showing no loss of conductivity over an 80 day test period (inset). The RS device I-II can be switched between the HRS and LRS using electrodes I-III (b, red trace). (d) High ON/OFF current ratios greater than $10^{5}$ are observed even while operating across electrodes I-III, where $V_{\text {READ }}=1 \mathrm{~V}$. Sweep rate was $50 \mathrm{mV} \mathrm{s}^{-1}$ for all data.

It is possible to drive the RS device defined by the I-II gap through the conducting network established within the II-III gap. With the RS device in the HRS state Fig. 8(b) (inset: red dash trace) shows that a $V_{\text {SET }}$ of $11 \mathrm{~V}$ applied across electrodes IIII switches the device into the LRS. Other than an increase in $V_{\text {RESET }}$ - corresponding to the addition of a load in the form of II-III - no change in RS parameters are seen. Fig. 8(d) shows that repeated cycling yields high ON/OFF ratios with welldefined current levels. Hence the same network can be programmed to act as a device channel (I-II) and an electrical interconnect material (II-III).

\section{Conclusions}

We have described the electrical characteristics of random nanowire networks with reversible junctions. We have shown that such networks exhibit properties that not only evolve and adapt in response to electrical stimuli leading to controlled levels of connectivity and conductivity, but that the incorporation of RS elements introduces a level of programmability not available in conventional materials. Networks are intrinsically self-programmable due to their capacity to dynamically reconfigure the distribution of ON and OFF junctions leading to fault-tolerant and adaptive behaviours. The natural length scales within networks defined by the nanowire dimensions, the size of connectivity cells and the separations between electrodes define a physical programmability whereby the properties of the confined network can be controlled and exploited for materials and device applications. The results presented here are transferable to other network systems, and thus provide a blueprint for the design of programmable materials from random percolative networks. Programmability will enable application in technologies where ease of fabrication, low cost, and maximum transparency/flexibility are a high priority such as artificial skin and sensing applications. The demonstrated ability to act as both device and interconnect suggests very low cost sensor technologies. Indeed further study of network materials will no doubt advance the art and provide additional opportunities for novel devices and sensors, including the possibility of networks that are responsive to non-electrical stimuli.

\section{Acknowledgements}

We acknowledge funding from the Science Foundation Ireland Investigator Programme (Grant no. 06/IN.1/I106) and a European Research Council Advanced Award (Grant no. AdG 321160). 


\section{References}

1 S. De, T. M. Higgins, P. E. Lyons, E. M. Doherty, P. N. Nirmalraj, W. J. Blau, J. J. Boland and J. N. Coleman, ACS Nano, 2009, 3, 1767-1774.

2 W. Gaynor, J.-Y. Lee and P. Peumans, ACS Nano, 2009, 4, 3034.

3 D. J. Lipomi, M. Vosgueritchian, B. C. K. Tee, S. L. Hellstrom, J. A. Lee, C. H. Fox and Z. N. Bao, Nat. Nanotechnol., 2011, 6, 788-792.

4 K. Takei, T. Takahashi, J. C. Ho, H. Ko, A. G. Gillies, P. W. Leu, R. S. Fearing and A. Javey, Nat. Mater., 2010, 9, 821-826.

5 Y. Weizmann, D. M. Chenoweth and T. M. Swager, J. Am. Chem. Soc., 2011, 133, 3238-3241.

6 P. Serre, C. Ternon, V. Stambouli, P. Periwal and T. Baron, Sens. Actuators, B, 2013, 182, 390-395.

7 L. B. Hu, H. S. Kim, J. Y. Lee, P. Peumans and Y. Cui, ACS Nano, 2010, 4, 2955-2963.

8 D. Langley, G. Giusti, C. Mayousse, C. Celle, D. Bellet and J.-P. Simonato, Nanotechnology, 2013, 24, 452001.

9 P. E. Lyons, S. De, J. Elias, M. Schamel, L. Philippe, A. T. Bellew, J. J. Boland and J. N. Coleman, J. Phys. Chem. Lett., 2011, 2, 3058-3062.

10 A. Sánchez-Iglesias, B. Rivas-Murias, M. Grzelczak, J. PérezJuste, L. M. Liz-Marzán, F. Rivadulla and M. A. CorreaDuarte, Nano Lett., 2012, 12, 6066-6070.

11 A. Morag, V. Ezersky, N. Froumin, D. Mogiliansky and R. Jelinek, Chem. Commun., 2013, 49, 8552-8554.

12 A. R. Rathmell, M. Nguyen, M. F. Chi and B. J. Wiley, Nano Lett., 2012, 12, 3193-3199.

13 P. N. Nirmalraj, A. T. Bellew, A. P. Bell, J. A. Fairfield, E. K. McCarthy, C. O'Kelly, L. F. C. Pereira, S. Sorel, D. Morosan, J. N. Coleman, M. S. Ferreira and J. J. Boland, Nano Lett., 2012, 12, 5966-5971.

14 R. Waser and M. Aono, Nat. Mater., 2007, 6, 833-840.

15 R. Waser, R. Dittmann, G. Staikov and K. Szot, Adv. Mater., 2009, 21, 2632-2663.

16 D. Ielmini, R. Bruchhaus and R. Waser, Phase Transitions, 2011, 84, 570-602.

17 R. Waser, J. Nanosci. Nanotechnol., 2012, 12, 7628-7640.
18 International Technology Roadmap for Semiconductors, http://www.itrs.net/.

19 T. Ohno, T. Hasegawa, T. Tsuruoka, K. Terabe, J. K. Gimzewski and M. Aono, Nat. Mater., 2011, 10, 591-595.

20 A. Z. Stieg, A. V. Avizienis, H. O. Sillin, C. Martin-Olmos, M. Aono and J. K. Gimzewski, Adv. Mater., 2012, 24, 286-293.

21 A. V. Avizienis, H. O. Sillin, C. Martin-Olmos, H. H. Shieh, M. Aono, A. Z. Stieg and J. K. Gimzewski, PLoS One, 2012, 7, e42772.

22 H. O. Sillin, E. J. Sandouk, A. V. Avizienis, M. Aono, A. Z. Stieg and J. K. Gimzewski, J. Nanosci. Nanotechnol., 2014, 14, 27922798.

23 A. Z. Stieg, A. V. Avizienis, H. O. Sillin, C. Martin-Olmos, M.-L. Lam, M. Aono and J. K. Gimzewski, Jpn. J. Appl. Phys., 2014, 53, 01AA02.

24 J. F. Gibbons and W. E. Beadle, Solid-State Electron., 1964, 7, 785-790.

25 S. Seo, M. J. Lee, D. H. Seo, E. J. Jeoung, D. S. Suh, Y. S. Joung, I. K. Yoo, I. R. Hwang, S. H. Kim, I. S. Byun, J. S. Kim, J. S. Choi and B. H. Park, Appl. Phys. Lett., 2004, 85, 56555657.

26 L. He, Z.-M. Liao, H.-C. Wu, X.-X. Tian, D.-S. Xu, G. L. W. Cross, G. S. Duesberg, I. V. Shvets and D.-P. Yu, Nano Lett., 2011, 11, 4601-4606.

27 C. Cagli, F. Nardi, B. Harteneck, Z. K. Tan, Y. G. Zhang and D. Ielmini, Small, 2011, 7, 2899-2905.

28 V. Scardaci, R. Coull, P. E. Lyons, D. Rickard and J. N. Coleman, Small, 2011, 7, 2621-2628.

29 U. Russo, D. Ielmini, C. Cagli and A. L. Lacaita, IEEE Trans. Electron Devices, 2009, 56, 193-200.

30 K. M. Kim, D. S. Jeong and C. S. Hwang, Nanotechnology, 2011, 22, 254002.

31 Y. Chen, J. Kang, B. Chen, B. Gao, L. Liu, X. Liu, Y. Wang, L. Wu, H. Yu and J. Wang, J. Phys. D: Appl. Phys., 2012, 45, 065303.

32 D. Adler, M. S. Shur, M. Silver and S. R. Ovshinsky, J. Appl. Phys., 1980, 51, 3289-3309.

33 I. Salaoru, A. Khiat, Q. Li, R. Berdan, C. Papavassiliou and T. Prodromakis, J. Phys. D: Appl. Phys., 2014, 47, 145102.

34 S. C. Chae, J. S. Lee, S. Kim, S. B. Lee, S. H. Chang, C. Liu, B. Kahng, H. Shin, D.-W. Kim, C. U. Jung, S. Seo, M.-J. Lee and T. W. Noh, Adv. Mater., 2008, 20, 1154-1159. 